Publ. RIMS, Kyoto Univ.

22 (1986), 81-95

\title{
On the Stable Homotopy of the Real Projective Space of Even Low Dimension
}

\author{
Dedicated to Professor Itiro Tamura on his 60th birthday \\ By \\ Juno MUKAI*
}

\section{§ 0. Introduction}

We denote by $\{X, Y\}$ the group of stable homotopy classes of mappings from $X$ to $Y$. We denote by $P^{n}$ the real $n$-dimensional projective space. The purpose of this note is to determine the group structure of $\left\{P^{2 n}, P^{2 n}\right\}$ for $2 \leqq n \leqq 4$ (Theorems 2.4, 3.4 and 5.6). As an application, the stable group of self-homotopy equivalences of $P^{2 n}$ will be determined in our case (Corollaries to the above theorems).

We denote by $\gamma_{n}: S^{n} \rightarrow P^{n}$ the projection. Let $\bar{\gamma}_{2 n}: E^{2 n-1} P^{2} \rightarrow P^{2 n}$ be a stable extension of $\gamma_{2 n}$ such that $P^{2 n+2}$ is the mapping cone of $\bar{\gamma}_{2 n}$. Then our method is to use the cofibre sequence starting with $\bar{\gamma}_{2 n}$ and to use the following: The order of the identity class of $P^{2 n}$ [11], the order of the Kahn-Priddy map [6] and the ring structure for $k \leqq 8$ of the stable homotopy ring of spheres $\pi_{*}=\sum \pi_{k}\left(S^{0}\right)$ [10]. The EHP-sequence is used to show that the generator $\sigma$ of the 2-component of $\pi_{7}\left(S^{0}\right)$ survives in $\left\{P^{8}, P^{4}\right\}$ (Lemma 5.2).

\section{§1. Main Results Used in the Computations}

Throughout this note we work in the stable category, unless otherwise stated. First we shall give a remark about the stable secondary compositions. The last part of Chap. III of [10] deals with them in the only case of the stable homotopy groups of spheres. But the definition of the stable secondary composition is still valid in the case of the stable homotopy groups between finite CW-complexes. The properties (3.5), (3.6), (3.7) and (3.8) of [10] are valid in our case. For example, we have the following:

$$
\alpha \circ\langle\beta, \gamma, \delta\rangle \subset(-1)^{|a|}\langle\alpha \circ \beta, \gamma, \delta\rangle
$$

Communicated by N. Shimada, June 27, 1985.

* Department of Mathematics, Faculty of Liberal Arts, Shinshu University, Matsumoto 390, Japan. 
and

$$
\langle\alpha, \beta, \gamma\rangle \circ \delta=(-1)^{\mid \alpha_{1}+1} \alpha \circ\langle\beta, \gamma, \delta\rangle,
$$

where $|\alpha|=\operatorname{dim} Y-\operatorname{dim} Z$ for $\alpha \in\{Y, Z\}$.

These properties of the stable secondary compositions will be freely used in the subsequent arguments.

We denote by $s(n)$ the number of $i$ such that $0<i \leqq n$ and $i \equiv 0,1,2$ or 4 mod 8. By Theorem 6.2 of [1], $P^{n-1}$ is reducible if and only if $2^{s(n-1)}$ is a divisor of $n$. So we have the following

Theorem 1.1. $\gamma_{2 n}$ is trivial if and only if $n=1$ or 3 .

It is well known that $2 \gamma_{2 n}=0$.

We denote by, the identity class of $S^{0}$, by $i: S^{1} \rightarrow P^{2}$ and $p: P^{2} \rightarrow S^{2}$ the canonical maps. Then we have a cofire sequence

$$
S^{1} \stackrel{2 \iota}{\longrightarrow} S^{1} \stackrel{i}{\longrightarrow} P^{2} \stackrel{p}{\longrightarrow} S^{2} \longrightarrow \cdots \text {. }
$$

We take $\bar{\gamma}_{2 n} \in\left\langle\gamma_{2 n}, 2 \iota, p\right\rangle$ such that $P^{2 n+2}$ is its mapping cone. Exactly we have a cofibre sequence

$$
E^{2 n-1} P^{2} \stackrel{\bar{\gamma}_{2 n}}{\longrightarrow} P^{2 n} \stackrel{i^{(n)}}{\longrightarrow} P^{2 n+2} \stackrel{p^{(n)}}{\longrightarrow} E^{2 n} P^{2} \longrightarrow \cdots,
$$

where $i^{(n)}$ and $p^{(n)}$ are the canonical maps.

Let $\eta$ be the generator of $\pi_{1}\left(S^{0}\right) \approx Z / 2$ and $\iota_{n}^{\prime}$ the identity class of $P^{n}$ Then, by [11], we have the following

Theorem 1.2. i) $\iota_{2 n}^{\prime}$ is of order $2^{s(2 n)}$.

ii) $2 \iota_{2}^{\prime}=i \eta p$.

A mapping $\phi: P^{2 n} \rightarrow S^{0}$ is called a Kahn-Priddy map if the restriction $\phi \mid S^{1}=\eta$. We denote by $\xi(X)$ the stable group of self-homotopy equivalences of $X$. Then, by Theorems 1.1 and 3.1 of [6], we have the following

Theorem 1.3. Let $\phi_{2 n}: P^{2 n} \rightarrow S^{0}$ be a Kahn-Priddy map. Then

i) $\phi_{2 n}$ is of order $2^{s(2 n)}$.

ii) There exists an element $\varepsilon_{2 n}^{\prime} \in \xi\left(P^{2 n}\right)$ such that $\left\langle\phi_{2 n}{ }^{\circ} \varepsilon_{2 n}^{\prime}, \bar{\gamma}_{2 n}, p^{(n)}\right\rangle$ contains a Kahn-Priddy map from $P^{2 n+2}$ to $S^{0}$.

This theorem will be used putting $\varepsilon_{2 n}^{\prime}=\ell_{2 n}^{\prime}$ in our arguments.

We shall use the following [10]

Theorem 1.4. i) $\pi_{k}\left(S^{0}\right)$ for $0 \leqq k \leqq 8$ (the 2-component for $k=3$ or 7 ) is isomorphic to the corresponding group in the following table: 


\begin{tabular}{|c|c|c|c|c|c|c|c|c|}
\hline$k$ & 0 & 1 & 2 & 3 & 4,5 & 6 & 7 & 8 \\
\hline$\pi_{k}\left(S^{0}\right)$ & $(\infty)$ & $(2)$ & $(2)$ & $(8)$ & 0 & $(2)$ & $(16)$ & $(2)^{2}$ \\
\hline gen. & $\iota$ & $\eta$ & $\eta^{2}$ & $\nu$ & & $\nu^{2}$ & $\sigma$ & $\eta \sigma, \varepsilon$ \\
\hline
\end{tabular}

Here $(n)$ means $Z / n$ and $(2)^{2}=(2) \oplus(2)$ (direct summand).

ii) There exist the following relations:

$$
\begin{aligned}
& \eta^{2}=\langle 2 \iota, \eta, 2 \iota\rangle, \quad \eta^{3}=4 \nu, \quad \eta \nu=\nu \eta=0, \quad 2 \nu \in\langle\eta, 2 \iota, \eta\rangle \bmod 4 \nu, \\
& \nu^{2}=\langle\eta, \nu, \eta\rangle, \quad \eta \sigma=\sigma \eta, \quad \varepsilon \in\left\langle\eta, 2 \iota, \nu^{2}\right\rangle=\langle\eta, \nu, 2 \nu\rangle \bmod \eta \sigma, \\
& \eta \sigma+\varepsilon=\langle\nu, \eta, \nu\rangle .
\end{aligned}
$$

By use of (1.1) and Theorem 1.4, we have the following (Theorems 3.1 and 3.2 of [4] and $\S 2$ of [5])

Theorem 1.5. i) $G_{k}=\pi^{0}\left(E^{k-2} P^{2}\right)$ and $G_{k}^{*}=\pi_{k+1}\left(P^{2}\right)$ are for $0 \leqq k \leqq 8$ isomorphic to the corresponding group in the following table:

\begin{tabular}{|c|c|c|c|c|c|c|c|c|c|}
\hline$k$ & 0 & 1 & 2 & 3 & 4 & 5 & 6 & 7 & 8 \\
\hline$G_{k} \approx G_{k}^{*}$ & $(2)$ & $(2)$ & $(4)$ & $(2)^{2}$ & $(2)$ & 0 & $(2)$ & $(2)^{2}$ & $(2)^{3}$ \\
\hline gen. of $G_{k}$ & $p$ & $\eta p$ & $\bar{\eta}$ & $\bar{\eta}, \nu p$ & $\eta^{2} \bar{\eta}$ & & $\nu^{2} p$ & $\overline{\nu^{2}}, \sigma p$ & $\overline{8 \sigma}, \eta \sigma p, \varepsilon p$ \\
\hline gen. of $G_{k}^{*}$ & $i$ & $i \eta$ & $\tilde{\eta}$ & $\tilde{\eta} \eta, i \nu$ & $\tilde{\eta} \eta^{2}$ & & $i \nu^{2}$ & $\widetilde{\nu^{2}}, i \sigma$ & $\overline{8 \sigma}, i \eta \sigma, i \varepsilon$ \\
\hline
\end{tabular}

ii) There exist the following relations:

$$
\begin{aligned}
& p i=0, \quad \iota \in\langle p, i, 2 \iota\rangle=\langle 2 \iota, p, i\rangle \bmod 2 \iota, \quad \bar{\eta} \in\langle\eta, 2 \iota, p\rangle, \quad \tilde{\eta} \in\langle i, 2 \iota, \eta\rangle, \\
& 2 \bar{\eta}=\eta^{2} p, \quad 2 \tilde{\eta}=i \eta^{2}, \quad \bar{\eta} \tilde{\eta}= \pm 2 \nu, \quad \nu \bar{\eta}=0, \quad \tilde{\eta} \nu=0, \quad \overline{\nu^{2}} \in\left\langle\nu^{2}, 2 \iota, p\right\rangle, \\
& \widetilde{\nu^{2}} \in\left\langle i, 2 \iota, \nu^{2}\right\rangle, \quad \eta \overline{\nu^{2}}=\varepsilon p, \quad \widetilde{\nu^{2}} \eta=i \varepsilon, \quad \overline{8 \sigma} \in\langle 8 \sigma, 2 \iota, p\rangle, \quad \widetilde{8 \sigma} \in\langle i, 2 \iota, 8 \sigma\rangle, \\
& \bar{\eta} \widetilde{\nu^{2}}=\overline{\nu^{2}} \tilde{\eta}=\varepsilon .
\end{aligned}
$$

By Theorem 3.3 of [4] and by Proposition 2.1 of [5], we have the following

Theorem 1.6. i) $H_{k}=\left\{E^{k} P^{2}, P^{2}\right\}$ for $-1 \leqq k \leqq 6$ is isomorphic to the corresponding group in the following table:

\begin{tabular}{|c|c|c|c|c|c|c|c|c|}
\hline$k$ & -1 & 0 & 1 & 2 & 3 & 4 & 5 & 6 \\
\hline$H_{k}$ & $(2)$ & $(4)$ & $(2)^{2}$ & $(2)^{3}$ & $(4) \oplus(2)$ & $(2)$ & $(2)$ & $(2)^{3}$ \\
\hline gen. & $i p$ & $\iota_{2}^{\prime}$ & $i \bar{\eta}, \tilde{\eta} p$ & $i \eta \bar{\eta}, \tilde{\eta} \eta p, i \nu p$ & $\tilde{\eta} \bar{\eta}, \overline{i \nu}$ & $\tilde{\eta} \eta \bar{\eta}$ & $\frac{i \nu^{2} p}{i \overline{\nu^{2}}, \widetilde{\nu^{2}} p, i \sigma p}$ \\
\hline
\end{tabular}

ii) There exist the following relations: 


$$
\begin{aligned}
& 2 \iota_{2}^{\prime}=i \eta p, \quad(i \bar{\eta})^{2}=i \eta \bar{\eta}, \quad(\tilde{\eta} p)^{2}=\tilde{\eta} \eta p, \quad \overline{i \nu} \in\langle i \nu, 2 \iota, p\rangle=\langle i, 2 \iota, \nu p\rangle \\
& \exists \widetilde{\nu p} \bmod 2 \tilde{\eta} \bar{\eta}, \quad \tilde{\eta} p(\tilde{\eta} \eta \bar{\eta})=(\tilde{\eta} \eta \bar{\eta})(i \bar{\eta})=\tilde{\eta} \eta^{2} \bar{\eta}=0 .
\end{aligned}
$$

We shall give a proof of the last relation (cf. the proof of Proposition 2.1. vi) of $[5])$ :

$$
\begin{aligned}
& \tilde{\eta} \eta^{2} \bar{\eta}=\tilde{\eta}\langle 2 \iota, \eta, 2 \iota\rangle \bar{\eta} \subset\langle 2 \tilde{\eta}, \eta, 2 \bar{\eta}\rangle=\left\langle i \eta^{2}, \eta, \eta^{2} p\right\rangle \subset\left\langle i \eta, \eta^{3}, \eta p\right\rangle \\
& =\langle i \eta, 4 \nu, \eta p\rangle .
\end{aligned}
$$

On the other hand,

$$
\langle i \eta, 4 \nu, \eta p\rangle \supset\langle i \eta \nu, 4 \iota, \eta p\rangle=\langle 0,4 \iota, \eta p\rangle \ni 0 \bmod (i \eta) \pi^{0}\left(E^{3} P^{2}\right)+\pi_{6}\left(P^{2}\right)(\eta p)=0 .
$$

This completes the proof.

The theorems in this section will be often used without any references.

\section{§2. Determination of $\left\{P^{4}, P^{4}\right\}$}

Hereafter $Z / 2$ is taken as the coefficients group of the cohomology. Since $S q^{2}: \widetilde{H}^{2}\left(P^{4}\right) \rightarrow \widetilde{H}^{4}\left(P^{4}\right)$ is nontrivial, we have

$$
\bar{\gamma}_{2}=\tilde{\eta} p \text {. }
$$

By Theorem 1.3 and (2.1), we have a Kahn-Priddy map $\bar{\eta}^{\prime} \in \pi^{0}\left(P^{4}\right)$ of order 8 satisfying $\bar{\eta}^{\prime} i^{\prime}=\bar{\eta}$, i. e.,

$$
\bar{\eta}^{\prime} \in\left\langle\bar{\eta}, \tilde{\eta} p, p^{\prime}\right\rangle \text {. }
$$

Here $i^{\prime}=i^{(1)}$ and $p^{\prime}=p^{(1)}$ in $(1.2)^{\prime}$. So, by use of the exact sequence induced from $(1.2)^{\prime}$, we have $4 \bar{\eta}^{\prime}=\eta^{2} \bar{\eta} p^{\prime}$ and $\pi^{0}\left(P^{4}\right)=\left\{\bar{\eta}^{\prime}\right\} \approx Z / 8$.

We put $p_{2 n}=E^{2 n-2} p \circ p^{(n-1)}: P^{2 n} \rightarrow S^{2 n}$. Similarly as above, by use of $(1.2)^{\prime}$, we have the following

Proposition 2.1. $\pi^{k}\left(P^{4}\right)$ for $0 \leqq k \leqq 4$ is isomorphic to the corresponding group in the following table:

\begin{tabular}{|c||c|c|c|c|c|}
\hline$k$ & 0 & 1 & 2 & 3 & 4 \\
\hline$\pi^{k}\left(P^{4}\right)$ & $(8)$ & $(2)^{2}$ & $(2)$ & 0 & $(2)$ \\
\hline gen. & $\bar{\eta}^{\prime}$ & $\eta \bar{\eta} p^{\prime}, \nu p_{4}$ & $\bar{\eta} p^{\prime}$ & & $p_{4}$ \\
\hline
\end{tabular}

Here $4 \bar{\eta}^{\prime}=\eta^{2} \bar{\eta} p^{\prime}$

By use of $(1.2)^{\prime}$, we have a short exact sequence 


$$
0 \longrightarrow \underset{\prod}{\{\tilde{\eta}\}} \stackrel{i_{*}^{\prime}}{\longrightarrow} \pi_{3}\left(P^{4}\right) \stackrel{p_{*}^{\prime}}{\longrightarrow}\{i\} \rightarrow 0
$$

We define an element $\tilde{i} \in \pi_{3}\left(P^{4}\right)$ by $p^{\prime} \tilde{i}=i$, i. e.,

$$
\tilde{i} \in\left\langle i^{\prime}, \tilde{\eta} p, i\right\rangle \text {. }
$$

Then $2 \tilde{i} \in\left\langle\imath^{\prime}, \tilde{\eta} p, i\right\rangle \cdot 2 \iota=-i^{\prime}\langle\tilde{\eta} p, i, 2 \iota\rangle \supset i^{\prime} \tilde{\eta}\langle p, i, 2 \iota\rangle \ni i^{\prime} \tilde{\eta} \bmod 2 i^{\prime} \pi_{3}\left(P^{2}\right)=\left\{2 i^{\prime} \tilde{\eta}\right\}$. So we have $2 \tilde{i}= \pm i^{\prime} \tilde{\eta}$ and $\pi_{3}\left(P^{4}\right)=\{\tilde{i}\} \approx Z / 8$.

By the similar arguments to the above, we have the following

\section{Proposition 2.2.}

\begin{tabular}{|c||c|c|c|c|}
\hline$k$ & 0 & 1 & 2 & 3 \\
\hline$\pi_{k+3}\left(P^{4}\right)$ & $(8)$ & $(2)^{2}$ & $(2)$ & $(2)$ \\
\hline gen. & $\tilde{i}$ & $\tilde{i} \eta, i^{\prime} i \nu$ & $\tilde{i}^{2}$ & $\tilde{i} \nu$ \\
\hline
\end{tabular}

Here $2 \tilde{i}= \pm i^{\prime} \tilde{\eta}$.

Hereafter the inclusions $i, i^{\prime}, \cdots$ (resp. the projections $p, p^{\prime}, \cdots$ ) are often used to denote the compositions of the inclusions (resp. the projections), unless any confusion occurs.

By use of (1.1) and Proposition 2.2, we have the following

\section{Proposition 2.3.}

\begin{tabular}{|c|c|c|c|c|c|}
\hline$k$ & 0 & 1 & 2 & 3 & 4 \\
\hline$\left\{E^{k} P^{2}, P^{4}\right\}$ & $(4)$ & $(2)^{2}$ & $(2)^{3}$ & $(4) \oplus(2)$ & $(2)^{2}$ \\
\hline gen. & $i^{\prime}$ & $i \bar{\eta}, \tilde{i} p$ & $i \eta \bar{\eta}, \tilde{i} \eta p, i \nu p$ & $\tilde{i} \bar{\eta}, i^{\prime} \overline{i \nu}$ & $\tilde{i} \eta \bar{\eta}, \tilde{i} \nu p$ \\
\hline
\end{tabular}

Theorem 2.4. i) $4 \iota_{4}^{\prime} \equiv i \eta \bar{\eta} p^{\prime} \bmod i \nu p_{4}$.

ii) $\left\{P^{4}, P^{4}\right\}=\left\{\iota_{4}^{\prime}, i \nu p_{4}\right\} \approx Z / 8 \oplus Z / 2$.

Proof. Consider the following exact sequence induced from $(1.2)^{\prime}$ :

$$
\left\{P^{2}, P^{4}\right\} \stackrel{i^{\prime *}}{\longleftarrow}\left\{P^{4}, P^{4}\right\} \stackrel{p^{\prime *}}{\longleftarrow}\left\{E^{2} P^{2}, P^{4}\right\} \stackrel{(\tilde{\eta} p)^{*}}{\longleftarrow}\left\{E P^{2}, P^{4}\right\} .
$$

Then, $(\tilde{\eta} p)^{*}(i \bar{\eta})=0$ and by Proposition 2.3, $(\tilde{\eta} p)^{*}(\tilde{i} p)=\tilde{i} \eta p \neq 0$. So we have a short exact sequence

$$
0 \longleftarrow\left\{i^{\prime}\right\} \stackrel{i^{\prime *}}{\longleftarrow}\left\{P^{4}, P^{4}\right\} \stackrel{p^{*}}{\longleftarrow}\{i \eta \bar{\eta}, i \nu p\} \longleftarrow 0 .
$$


By (2.2) and Proposition 2.1, $\bar{\eta}^{\prime}\left(i \eta \bar{\eta} p^{\prime}\right)=\eta^{2} \bar{\eta} p^{\prime}=4 \bar{\eta}^{\prime}$ and $\bar{\eta}^{\prime}(i \nu p)=\eta \nu p=0$. This completes the proof.

We denote by $(Z / n)^{*}$ the multiplicative group of $Z / n$ and by $G \times H$ the direct product of groups $G$ and $H$. In the above theorem, $\left(i \nu p_{4}\right)^{2}=0$. So we have the following

Corollary。

$$
\xi\left(P^{4}\right) \approx(Z / 8) * \times Z / 2 \text {. }
$$

By (2.2) and (2.3), $2 \bar{\eta}^{\prime} \tilde{i} \in\langle\bar{\eta}, \tilde{\eta} p, i\rangle \cdot 2 \iota=-\bar{\eta}\langle\tilde{\eta} p, i, 2 \iota\rangle \supset \bar{\eta} \tilde{\eta}\langle p, i, 2 \iota\rangle \ni \pm 2 \nu$ $\bmod (2 \bar{\eta}) \pi_{3}\left(P^{2}\right)=\{4 \nu\}$. So we have

$$
\bar{\eta}^{\prime} \tilde{i} \equiv \nu \bmod 2 \nu \text {. }
$$

\section{§3. Determination of $\left\{P^{6}, P^{6}\right\}$}

Since $S q^{4}: \widetilde{H}^{1}\left(P^{5}\right) \rightarrow \widetilde{H}^{5}\left(P^{5}\right)$ is trivial, $\gamma_{4}=\tilde{i} \eta$ by Proposition 2.2. So, by Proposition 2.3 , we can take

$$
\bar{\gamma}_{4}=\tilde{i} \bar{\eta}
$$

By (2.4), $\bar{\eta}^{\prime}(\hat{i} \bar{\eta})=\nu \bar{\eta}=0$. So, by Theorem 1.3, we have a Kahn-Priddy map $\overline{\bar{\eta}} \in \pi^{0}\left(P^{6}\right)$ of order 8 satisfying $\overline{\bar{\eta}} i^{\prime \prime}=\bar{\eta}^{\prime}$, i.e.,

$$
\overline{\bar{\eta}} \in\left\langle\bar{\eta}^{\prime}, \tilde{i} \bar{\eta}, p^{\prime \prime}\right\rangle \text {. }
$$

By use of (1.2)" and (3.2), we have a split exact sequence

$$
0 \longleftarrow \underset{\prod}{\left\{\bar{\eta}^{\prime}\right\}} \underset{Z / 8}{\stackrel{i^{\prime \prime *}}{\longleftarrow}} \pi^{0}\left(P^{6}\right) \stackrel{p^{\prime \prime *}}{\longleftarrow}\left\{\nu^{2} p\right\} \longleftarrow 0 .
$$

Therefore $\pi^{0}\left(P^{6}\right)=\left\{\overline{\bar{\eta}}, \nu^{2} p_{6}\right\} \approx Z / 8 \oplus Z / 2$. i. e.,

By (2.3), $p_{4} \tilde{i}=p i=0$. So we can define an element $\bar{p}_{4} \in \pi^{4}\left(P^{6}\right)$ by $\bar{p}_{4} i^{\prime \prime}=p_{4}$,

$$
\bar{p}_{4} \in\left\langle p_{4}, \tilde{i} \bar{\eta}, p^{\prime \prime}\right\rangle
$$

Then, $2 \bar{p}_{4} \in 2 \iota \cdot\left\langle p_{4}, \tilde{i} \bar{\eta}, p^{\prime \prime}\right\rangle=-\left\langle 2 \iota, p_{4}, \tilde{i} \bar{\eta}\right\rangle p^{\prime \prime} \supset-\left\langle 2 \iota, p, p^{\prime} \tilde{i} \bar{\eta}\right\rangle p^{\prime \prime} \supset-\langle 2 \iota, p, i\rangle \bar{\eta} p^{\prime \prime} \ni$ $-\bar{\eta} p^{\prime \prime} \bmod 2 \pi^{0}\left(P^{2}\right) p^{\prime \prime}+\pi^{4}\left(E P^{4}\right)\left(\tilde{i} \bar{\eta} p^{\prime \prime}\right)=\left\{2 \bar{\eta} p^{\prime \prime}\right\}$ by Proposition 2.1. So we have $2 \bar{p}_{4}= \pm \bar{\eta} p^{\prime \prime}$. Therefore (1.2)" and Proposition 2.1 lead us to the following

Proposition 3.1. $\pi^{k}\left(P^{6}\right)$ for $0 \leqq k \leqq 6$ is isomorphic to the corresponding group in the following table: 


\begin{tabular}{|c||c|c|c|c|c|c|c|}
\hline$k$ & 0 & 1 & 2 & 3 & 4 & 5 & 6 \\
\hline$\pi^{k}\left(P^{6}\right)$ & $(8) \oplus(2)$ & $(2)$ & 0 & $(2)$ & $(8)$ & $(2)$ & $(2)$ \\
\hline gen. & $\overline{\bar{\eta}}, \nu^{2} p_{6}$ & $\nu \bar{p}_{4}$ & & $\nu p_{6}$ & $\bar{p}_{4}$ & $\eta p_{6}$ & $p_{6}$ \\
\hline
\end{tabular}

Here $2 \bar{p}_{4}= \pm \bar{\eta} p^{\prime \prime}$.

By use of (1.2)" and Proposition 2.2, we have the following

\section{Proposition 3.2.}

\begin{tabular}{|c||c|c|c|}
\hline$k$ & 0 & 1 & 2 \\
\hline$\pi_{k+4}\left(P^{6}\right)$ & $(2)$ & 0 & $(2)$ \\
\hline gen. & $i \nu$ & & $i^{\prime \prime} \tilde{i} \nu$ \\
\hline
\end{tabular}

By use of (1.2)" and Proposition 2.3, we have the following

\section{Proposition 3.3.}

\begin{tabular}{|c||c|c|c|}
\hline$k$ & 2 & 3 & 4 \\
\hline$\left\{E^{k} P^{2}, P^{6}\right\}$ & $(2)^{2}$ & $(2)$ & $(2)$ \\
\hline gen. & $i \eta \bar{\eta}, i \nu p$ & $i^{\prime} \overline{i \nu}$ & $i^{\prime \prime} \tilde{i} \nu p$ \\
\hline
\end{tabular}

Consider the following exact sequence:

$$
\pi^{4}\left(P^{4}\right) \stackrel{\gamma_{4 *}}{\longrightarrow}\left\{P^{4}, P^{4}\right\} \stackrel{i_{*}^{\prime \prime}}{\longrightarrow}\left\{P^{4}, P^{5}\right\} \longrightarrow 0 .
$$

Then, by $(1.2)^{\prime}, \gamma_{4} p_{4}=\tilde{i} \eta p p^{\prime}=p^{\prime *}(\tilde{\eta} p)^{*}(\tilde{i} p)=0$. So, by Theorem 2.4 , we have $\left\{P^{4}, P^{6}\right\}=\left\{i^{\prime \prime}, i \nu p_{4}\right\} \approx Z / 8 \oplus Z / 2$.

Theorem 3.4. $\left\{P^{6}, P^{6}\right\}=\left\{\iota_{6}^{\prime}, i \nu \bar{p}_{4}, i^{\prime \prime} \tilde{i \nu}_{\nu}\right\} \approx Z / 8 \oplus(Z / 2)^{2}$.

Proof. Consider the following exact sequence induced from (1.2)" :

$$
\left\{E^{3} P^{2}, P^{6}\right\} \stackrel{(i \bar{\eta})^{*}}{\longleftarrow}\left\{P^{4}, P^{6}\right\} \stackrel{i^{\prime \prime *}}{\longleftarrow}\left\{P^{6}, P^{6}\right\} \stackrel{p^{\prime \prime *}}{\longleftarrow}\left\{E^{4} P^{2}, P^{6}\right\} \stackrel{(\tilde{i} \bar{\eta})^{*}}{\longleftarrow}\left\{E P^{4}, P^{6}\right\} .
$$

Then $(\tilde{i} \bar{\eta}) *\left(i \nu p_{4}\right)=0$ and by Proposition 3.2, $(\tilde{i} \bar{\eta})^{*}\left\{E P^{4}, P^{6}\right\} \subset \pi_{4}\left(P^{6}\right) \bar{\eta}=0$. So we have a short exact sequence

$$
\begin{aligned}
& 0 \longleftarrow\left\{i^{\prime \prime}, i \nu p_{4}\right\} \stackrel{i^{\prime \prime *}}{\longleftarrow}\left\{P^{6}, P^{6}\right\} \stackrel{p^{\prime \prime *}}{\longleftarrow}\left\{i^{\prime \prime} \tilde{i}_{\eta} p\right\} \longleftarrow 0 . \\
& Z / 8 \oplus Z / 2 \quad Z / 2
\end{aligned}
$$


By (3.3), there exists an element $i \nu \bar{p}_{4}$ of order 2 in $\left\{P^{6}, P^{6}\right\}$. Since $\iota_{6}^{\prime}$ is of order 8 , the above sequence is split. This completes the proof.

We put $\alpha=i \nu \bar{p}_{4}$ and $\beta=i^{\prime \prime} i \nu p_{6}$. Then $\alpha^{2}=\beta^{2}=0$ and $\alpha \beta=\beta \alpha=0$. This leads us to the following

\section{Corollary. $\quad \xi\left(P^{6}\right) \approx(Z / 8)^{*} \times(Z / 2)^{2}$.}

By use of (1.1) and Proposition 3.1, we have the following

Proposition 3.5. $\left\{P^{6}, E^{k} P^{2}\right\}$ for $-1 \leqq k \leqq 4$ is isomorphic to the corresponding group in the following table:

\begin{tabular}{|c||c|c|c|c|c|c|}
\hline$k$ & -1 & 0 & 1 & 2 & 3 & 4 \\
\hline$\left\{P^{6}, E^{k} P^{2}\right\}$ & $(2)^{3}$ & $(2)$ & $(2)$ & $(2)^{2}$ & $(2)^{2}$ & $(4)$ \\
\hline gen. & $\widetilde{\nu \bar{p}_{4}, i \overline{\bar{\eta}}, i \nu^{2} p_{6}}$ & $i \nu \bar{p}_{4}$ & $\widetilde{\nu p p^{\prime \prime}}$ & $\tilde{\eta} \eta p_{6}, i \nu p_{6}$ & $\tilde{\eta} p_{6}, i \bar{p}_{4}$ & $p^{\prime \prime}$ \\
\hline
\end{tabular}

Here $\nu \widetilde{p}_{4} \in\left\langle i, 2 \iota, \nu \bar{p}_{4}\right\rangle$ and $2 p^{\prime \prime}=i \eta p_{6}$.

By use of $(1.2)^{\prime},(1.2)^{\prime \prime}$ and Proposition 3.5, we have the following

Proposition 3.6. i) $\left\{P^{6}, P^{4}\right\}=\left\{\widetilde{\tilde{\eta} \eta p_{6}}, \tilde{i} \nu p_{6}, i \nu \bar{p}_{4}\right\} \approx(Z / 2)^{3}$, where $\tilde{\eta} \eta p_{6} \in\left\langle i^{\prime}, \tilde{\eta} p, \tilde{\eta} \eta p_{6}\right\rangle$.

ii) $4 \iota_{6}^{\prime} \equiv i^{\prime \prime} \tilde{\eta} \eta p_{6} \bmod \left\{i^{\prime \prime} \tilde{i} p_{6}, i \nu \bar{p}_{4}\right\}$.

\section{§4. Determination of Generators of $\pi^{k}\left(P^{8}\right)$ for $0 \leqq k \leqq 8$}

By use of $(1.2)^{\prime}$, we have a short exact sequence

$$
0 \longrightarrow\left\{i \nu^{2}\right\} \stackrel{i_{*}^{\prime}}{\longrightarrow} \pi_{7}\left(P^{4}\right) \stackrel{p_{*}^{\prime}}{\rightarrow}\left\{\tilde{\eta} \eta^{2}\right\} \longrightarrow 0 .
$$

We define an element $\widetilde{\tilde{\eta} \eta^{2}} \in \pi_{7}\left(P^{4}\right)$ by $p^{\prime} \tilde{\eta} \eta^{2}=\tilde{\eta} \eta^{2}$, i. e.,

$$
\widetilde{\eta} \eta^{2} \in\left\langle i^{\prime}, \tilde{\eta} p, \tilde{\eta} \eta^{2}\right\rangle \text {. }
$$

By use of (1.2)", we have a short exact sequence

$$
0 \longrightarrow \pi_{7}\left(P^{4}\right) \stackrel{i_{*}^{\prime \prime}}{\longrightarrow} \pi_{7}\left(P^{6}\right) \stackrel{p_{*}^{\prime \prime}}{\longrightarrow}\{\tilde{\eta}\} \longrightarrow 0 .
$$

We define an element $\tilde{\eta}^{\prime} \in \pi_{7}\left(P^{6}\right)$ by $p^{\prime \prime} \tilde{\eta}^{\prime}=\tilde{\eta}$, i. e.,

$$
\tilde{\eta}^{\prime} \in\left\langle i^{\prime \prime}, \tilde{i} \bar{\eta}, \tilde{\eta}\right\rangle \text {. }
$$

By (3.3) and (4.2), $\left.\bar{p}_{4} \tilde{\eta}^{\prime} \in\left\langle p_{4}, \tilde{i} \bar{\eta}, \tilde{\eta}\right\rangle \subset\langle p, i \bar{\eta}, \tilde{\eta}\rangle \supset\langle p, i, \pm 2 \nu\rangle \supset\langle p, i, 2 \iota\rangle \cdot \pm \nu\right) \ni \pm \nu$ 
$\bmod p \pi_{5}\left(P^{2}\right)+\pi^{0}\left(P^{2}\right) \tilde{\eta}=\{2 \nu\}$. So we have

$$
\bar{p}_{4} \tilde{\eta}^{\prime} \equiv \nu \bmod 2 \nu \text {. }
$$

Proposition 4.1. i) $\tilde{\eta}^{\prime}$ is of order 8 .

ii) $4 \tilde{\eta}^{\prime} \equiv i^{\prime \prime} \tilde{\eta} \eta^{2} \bmod i \nu^{2}$.

iii) $\pi_{7}\left(P^{4}\right)=\left\{\widetilde{\eta} \eta^{2}, i \nu^{2}\right\} \approx(Z / 2)^{2}$.

iv) $\pi_{7}\left(P^{6}\right)=\left\{\tilde{\eta}^{\prime}, i \nu^{2}\right\} \approx Z / 8 \oplus Z / 2$.

Proof. i) follows from (4.3). By (4.2), $4 \tilde{\eta}^{\prime} \in\left\langle i^{\prime \prime}, \tilde{i} \bar{\eta}, \tilde{\eta}\right\rangle \circ 4 \iota=-i^{\prime \prime}\langle\tilde{i} \bar{\eta}, \tilde{\eta}, 4 \iota\rangle$. On the other hand, $p^{\prime}\langle\tilde{i} \bar{\eta}, \tilde{\eta}, 4 \iota\rangle \subset\langle i \bar{\eta}, \tilde{\eta}, 4 \iota\rangle \subset\langle i, \pm 2 \nu, 4 \iota\rangle \supset\langle i, 2 \iota, 4 \nu\rangle \ni \tilde{\eta} \eta^{2}$ $\bmod i \pi_{4}\left(S^{0}\right)+4 \pi_{5}\left(P^{2}\right)=0$. So we have $\langle\tilde{i} \bar{\eta}, \tilde{\eta}, 4 \iota\rangle \ni \tilde{\eta} \tilde{\eta}^{2} \bmod i \nu^{2}$. This leads us to ii). iii) and iv) follow from i) and ii). This completes the proof.

By Proposition 4.1, $\bar{\gamma}_{6} \in\left\langle\gamma_{6}, 2 \iota, p\right\rangle=\langle 0,2 \iota, p\rangle=\pi_{7}\left(P^{6}\right) p=\left\{\tilde{\eta}^{\prime} p, i \nu^{2} p\right\}$. By (4.2), Propositions 3.3 and 4.1, $\tilde{\eta}^{\prime} \in\left\langle i^{\prime \prime}, \tilde{i} \bar{\eta}, \tilde{\eta}\right\rangle \bmod i^{\prime \prime} \pi_{7}\left(P^{4}\right)+\left\{E^{4} P^{2}, P^{6}\right\} \tilde{\eta}=\left\{4 \tilde{\eta}^{\prime}, i \nu^{2}\right\}$. Since $S q^{2}: \widetilde{H}^{6}\left(P^{8}\right) \rightarrow \widetilde{H}^{8}\left(P^{8}\right)$ is nontrivial, we can take

$$
\bar{\gamma}_{6}=\tilde{\eta}^{\prime} p \text { for a suitable representative } \tilde{\eta}^{\prime} \in\left\langle i^{\prime \prime}, \tilde{i} \bar{\eta}, \tilde{\eta}\right\rangle \text {. }
$$

By use of (1.2)", we have a short exact sequence

$$
0 \longrightarrow \pi_{7}\left(P^{6}\right) \stackrel{i_{*}^{\prime \prime \prime}}{\longrightarrow} \pi_{7}\left(P^{8}\right) \stackrel{p_{*}^{\prime \prime \prime}}{\longrightarrow}\{i\} \longrightarrow 0 .
$$

We define an element $\tilde{i}^{\prime} \in \pi_{7}\left(P^{8}\right)$ by $p^{\prime \prime \prime} i^{\prime}=i$, i. e.,

$$
\tilde{i}^{\prime} \in\left\langle i^{\prime \prime \prime}, \tilde{\eta}^{\prime} p, i\right\rangle \text {. }
$$

Then $2 \tilde{i}^{\prime} \in\left\langle i^{\prime \prime \prime}, \tilde{\eta}^{\prime} p, i\right\rangle \cdot 2 \iota=-i^{\prime \prime \prime}\left\langle\tilde{\eta}^{\prime} p, i, 2 \iota\right\rangle \ni i^{\prime \prime \prime} \tilde{\eta}^{\prime} \bmod 2 i_{*}^{\prime \prime \prime} \pi_{7}\left(P^{6}\right)=\left\{2 i^{\prime \prime \prime} \tilde{\eta}^{\prime}\right\}$ by Proposition 4.1. This leads us to the following

Proposition 4.2. $2 \tilde{i}^{\prime \prime} \equiv i^{\prime \prime \prime} \tilde{\eta}^{\prime} \bmod 2 i^{\prime \prime \prime} \tilde{\eta}^{\prime}$ and $\pi_{7}\left(P^{8}\right)=\left\{\tilde{i}^{\prime}, i \nu^{2}\right\} \approx Z / 16 \oplus Z / 2$ 。

By use of $(1.2)^{(n)}$ for $n=1,2$ and 3 , we have the following

Proposition 4.3. $\pi_{8}\left(P^{2 k}\right)$ for $2 \leqq k \leqq 4$ is isomorphic to the corresponding group in the following table:

\begin{tabular}{|c||c|c|c|}
\hline$k$ & 2 & 3 & 4 \\
\hline$\pi_{8}\left(P^{2 k}\right)$ & $(2)^{2}$ & $(2)^{4}$ & $(2)^{4}$ \\
\hline gen. & $i^{\prime} \widetilde{\nu}^{2}, i \sigma$ & $\tilde{\eta}^{\prime} \eta, \widetilde{i \nu}, i^{\prime} \nu^{2}, i \sigma$ & $\tilde{i}^{\prime} \eta, i^{\prime \prime \prime} \widetilde{i \nu}, i^{\prime} \widetilde{\nu}^{2}, i \sigma$ \\
\hline
\end{tabular}

Here $\tilde{i \nu} \in\left\langle i^{\prime \prime}, \tilde{i} \bar{\eta}, i \nu\right\rangle$.

Since $S q^{2}: \widetilde{H}^{7}\left(P^{9}\right) \rightarrow \widetilde{H}^{9}\left(P^{9}\right)$ is nontrivial and $S q^{8}: \widetilde{H}^{1}\left(P^{9}\right) \rightarrow \widetilde{H}^{9}\left(P^{9}\right)$ is trivial, 
$\gamma_{8} \equiv \tilde{i}^{\prime} \eta \bmod \left\{i^{\prime \prime \prime} \widetilde{i \nu}, i^{\prime} \widetilde{\nu^{2}}\right\}$ by Proposition 4.3. So we have the following

Proposition 4.4. i) $\gamma_{8} \equiv \tilde{i}^{\prime} \eta \bmod \left\{i^{\prime \prime \prime} \tilde{i \nu}, i^{\prime} \nu^{2}\right\}$.

ii) $\pi_{8}\left(P^{9}\right)=\left\{i^{\prime \prime \prime} \widetilde{i \nu}, i^{\prime} \nu^{2}, i \sigma\right\} \approx(Z / 2)^{3}$.

Remark 1. By Proposition 3.2, $\pi_{6}\left(P^{8}\right)=\left\{i^{\prime \prime} i \nu\right\} \approx Z / 2$. So Propositions 2.2, 3.2, 4.2 and 4.4 overlap with Theorem 2.6 of [2] and Table 4.1 of [3].

Next we shall determine generators of $\pi^{k}\left(P^{8}\right)$ for $0 \leqq k \leqq 8$. We define an element $\overline{\nu p} \in \pi^{0}\left(E P^{4}\right)$ by $\overline{\nu p} i^{\prime}=\nu p$, i. e., $\overline{\nu p} \in\left\langle\nu p, \tilde{\eta} p, p^{\prime}\right\rangle$. Then, by use of $(1.2)^{\prime}$, we have $\pi^{0}\left(E P^{4}\right)=\left\{\eta \bar{\eta}^{\prime}, \overline{\nu p}\right\} \approx(Z / 2)^{2}$.

Consider the following exact sequence induced from (1.2)" :

$$
\pi^{0}\left(E^{4} P^{2}\right) \stackrel{(\tilde{i} \bar{\eta})^{*}}{\longleftarrow} \pi^{0}\left(E P^{4}\right) \stackrel{i^{\prime \prime *}}{\longleftarrow} \pi^{0}\left(E P^{6}\right) \stackrel{p^{\prime \prime *}}{\longleftarrow} \pi^{0}\left(E^{5} P^{2}\right) \stackrel{(\tilde{i} \bar{\eta})^{*}}{\longleftarrow} \pi^{0}\left(E^{2} P^{4}\right) .
$$

Then $(\tilde{i} \bar{\eta}) * \pi^{0}\left(E^{k} P^{4}\right) \subset \pi_{k+3}\left(S^{0}\right) \bar{\eta}=0$ for $k=1$ or 2 , and so we have a short exact sequence

$$
\begin{aligned}
& 0 \longleftarrow\left\{\eta \bar{\eta}^{\prime}, \overline{\nu p}\right\} \stackrel{i^{\prime \prime *}}{\longleftarrow} \pi^{0}\left(E P^{6}\right) \stackrel{p^{\prime \prime *}}{\longleftarrow}\left\{\overline{\nu^{2}}, \sigma p\right\} \longleftarrow 0 . \\
& (Z / 2)^{2} \quad(Z / 2)^{2}
\end{aligned}
$$

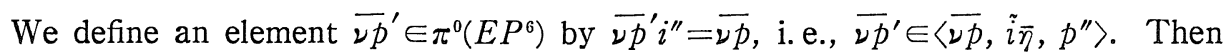
$2 \overline{\nu p}^{\prime} \in 2 \iota\left\langle\overline{\nu p}, \tilde{i} \bar{\eta}, p^{\prime \prime}\right\rangle=-\langle 2 \iota, \overline{\nu p}, \tilde{i} \bar{\eta}\rangle p^{\prime \prime} \subset-\langle 2 \iota, 0, \bar{\eta}\rangle p^{\prime \prime}=2 \pi^{0}\left(E^{5} P^{2}\right) p^{\prime \prime}+\pi_{5}\left(S^{0}\right) \bar{\eta} p^{\prime \prime}$ $=0$. So, by (3.2), we have the following

Proposition 4.5. $\pi^{0}\left(E P^{6}\right)=\left\{\eta \bar{\eta}, \overline{\nu p^{\prime}}, \overline{\nu^{2}} p^{\prime \prime}, \sigma p_{6}\right\} \approx(Z / 2)^{4}$.

Consider the following exact sequence induced from $(1.2)^{\prime \prime \prime}$ :

$$
\pi^{0}\left(E^{5} P^{2}\right) \stackrel{\left(\tilde{\eta}^{\prime} p\right)^{*}}{\longleftarrow} \pi^{0}\left(P^{6}\right) \stackrel{i i^{\prime \prime *}}{\longleftarrow} \pi^{0}\left(P^{8}\right) \stackrel{p^{\prime \prime \prime *}}{\longleftarrow} \pi^{0}\left(E^{6} P^{2}\right) \stackrel{\left(\tilde{\eta}^{\prime} p\right)^{*}}{\longleftarrow} \pi^{0}\left(E P^{6}\right) .
$$

Then, by Proposition 3.1, $\left(\tilde{\eta}^{\prime} p\right)^{*}\left(\nu^{2} p_{6}\right)=0$ and $\left(\tilde{\eta}^{\prime} p\right)^{*} \overline{\bar{\eta}}=\overline{\bar{\eta}} \tilde{\eta}^{\prime} p$. Since $\overline{\bar{\eta}}$ is of order $8, \bar{\eta} \tilde{\eta}^{\prime}=2 a \sigma$ for some integer $a$. So the first $\left(\tilde{\eta}^{\prime} p\right)^{*}$ is trivial. By (4.2) and Proposition 4.5, $\quad\left(\tilde{\eta}^{\prime} p\right) *(\eta \bar{\eta})=0, \quad\left(\tilde{\eta}^{\prime} p\right)^{*}\left(\overline{\nu^{2}} p^{\prime \prime}\right)=\varepsilon p, \quad\left(\tilde{\eta}^{\prime} p\right)^{*}\left(\sigma p_{6}\right)=\sigma \eta p \quad$ and $\left(\tilde{\eta}^{\prime} p\right)^{*} \overline{\nu p^{\prime}} \in \pi_{8}\left(S^{0}\right) p$. Therefore we have

$$
\pi_{8}\left(S^{0}\right) p_{8}=0
$$

and a short exact sequence

$$
\begin{aligned}
& 0 \longleftarrow\left\{\overline{\bar{\eta}}, \nu^{2} p_{6}\right\} \stackrel{i^{\prime \prime \prime *}}{\longleftarrow} \pi^{0}\left(P^{8}\right) \stackrel{p^{\prime \prime \prime * *}}{\longleftarrow}\{\overline{8 \sigma}\} \longleftarrow 0 . \\
& Z / 8 \oplus Z / 2 \quad Z / 2
\end{aligned}
$$

We define an element $\overline{\bar{\nu}}_{6} \in \pi^{3}\left(P^{8}\right)$ by $\overline{\overline{\nu p}}_{6} i^{\prime \prime \prime}=\nu p_{6}$, i.e., 


$$
\overline{\overline{\nu p}}_{6} \in\left\langle\nu p_{6}, \tilde{\eta}^{\prime} p, p^{\prime \prime \prime}\right\rangle \text {. }
$$

By (4.6) and (4.7), $2 \nu \nu \overline{\bar{p}}_{6} \in 2 \nu \circ\left\langle\nu p_{6}, \tilde{\eta}^{\prime} p, p^{\prime \prime \prime}\right\rangle=\left\langle 2 \nu, \nu p_{6}, \tilde{\eta}^{\prime} p\right\rangle p^{\prime \prime \prime} \supset\langle 2 \nu, \nu, \eta p\rangle p^{\prime \prime \prime} \supset$ $\langle 2 \nu, \nu, \eta\rangle p p^{\prime \prime \prime}=0 \bmod 2 \nu \pi^{0}\left(E^{3} P^{2}\right)=0$. So we have $2 \nu \overline{\bar{p}}_{6}=0$. By Theorem 1.3, we have a Kahn-Priddy map $\overline{\bar{\eta}}^{\prime} \in \pi^{0}\left(P^{8}\right)$ of order 16 satisfying $\overline{\bar{\eta}}^{\prime} i^{\prime \prime \prime}=\overline{\bar{\eta}}$, i. e.,

$$
\overline{\bar{\eta}}^{\prime} \in\left\langle\overline{\bar{\eta}}, \tilde{\eta}^{\prime} p, p^{\prime \prime \prime}\right\rangle \text {. }
$$

Therefore we have the following

Theorem 4.6. $8 \overline{\bar{\eta}}^{\prime}=\overline{8 \sigma} p^{\prime \prime \prime}$ and $\pi^{0}\left(P^{8}\right)=\left\{\overline{\bar{\eta}}^{\prime}, \nu \overline{\bar{p}}_{6}\right\} \approx Z / 16 \oplus Z / 2$.

By (4.6) and (4.8), $8 \overline{\bar{\eta}}^{\prime} \in 8 \iota \cdot\left\langle\overline{\bar{\eta}}, \tilde{\eta}^{\prime} p, p^{\prime \prime \prime}\right\rangle=-\left\langle 8 \iota, \overline{\bar{\eta}}, \tilde{\eta}^{\prime} p\right\rangle p^{\prime \prime \prime} \subset-\langle 8 \iota, 2 a \sigma, p\rangle p^{\prime \prime \prime}$ $\supset-a\langle 8 \sigma, 2 \iota, p\rangle p^{\prime \prime \prime} \ni a \overline{8 \sigma} p^{\prime \prime \prime} \bmod 8 \pi^{0}\left(E^{6} P^{2}\right) p^{\prime \prime \prime}+\pi_{8}\left(S^{0}\right) p_{8}=0$. So, by Theorem 4.6, $a$ must be odd and we have

$$
\overline{\bar{\eta}} \tilde{\eta}^{\prime} \equiv 2 \sigma \bmod 4 \sigma .
$$

Consider the following exact sequence induced from $(1.2)^{\prime \prime \prime}$ :

$$
\pi^{0}\left(E P^{2}\right) \stackrel{\left(\tilde{\eta}^{\prime} p\right)^{*}}{\longleftarrow} \pi^{4}\left(P^{6}\right) \stackrel{i^{\prime \prime *} *}{\longleftarrow} \pi^{4}\left(P^{8}\right) \stackrel{p^{\prime \prime \prime * *}}{\longleftarrow} \pi^{0}\left(E^{2} P^{2}\right) \stackrel{\left(\tilde{\eta}^{\prime} p\right)^{*}}{\longleftarrow} \pi^{3}\left(P^{6}\right) .
$$

Then, by Proposition 3.1 and $(4.3),\left(\tilde{\eta}^{\prime} p\right) *\left(\nu p_{6}\right)=0,\left(\tilde{\eta}^{\prime} p\right)^{*} \bar{p}_{4}=\nu p$ and $\operatorname{Im} i^{\prime \prime \prime *}=$ $\left\{\bar{\eta} p^{\prime \prime}\right\} \approx Z / 4$. So we have a short exact sequence

$$
\begin{aligned}
& 0 \longleftarrow\left\{\bar{\eta} p_{\eta}\right\} \stackrel{i^{\prime \prime \prime * *}}{\longleftarrow} \pi^{4}\left(P^{8}\right) \stackrel{p^{\prime \prime \prime *}}{\longleftarrow}\left\{\eta_{\eta}^{2} \bar{\eta}\right\} \longleftarrow 0 . \\
& Z / 4 \quad Z / 2
\end{aligned}
$$

We define an element $\overline{\bar{\eta} p^{\prime \prime}} \in \pi^{4}\left(P^{8}\right)$ by $\overline{\bar{\eta} p^{\prime \prime}} i^{\prime \prime \prime}=\bar{\eta} p^{\prime \prime}$, i. e., $\overline{\bar{\eta} p^{\prime \prime}} \in\left\langle\bar{\eta} p^{\prime \prime}, \tilde{\eta}^{\prime} p, p^{\prime \prime \prime}\right\rangle$. Then $4 \overline{\bar{\eta} p^{\prime \prime}} \in 4 \iota \cdot\left\langle\bar{\eta} p^{\prime \prime}, \tilde{\eta}^{\prime} p, p^{\prime \prime \prime}\right\rangle=-\left\langle 4 \iota, \bar{\eta} p^{\prime \prime}, \tilde{\eta}^{\prime} p\right\rangle p^{\prime \prime \prime} \subset-\langle 4 \iota, \pm 2 \nu, p\rangle p^{\prime \prime \prime} \supset-\langle 4 \nu, 2 \iota$, $p\rangle p^{\prime \prime \prime} \ni \eta^{2} \bar{\eta} p^{\prime \prime \prime} \bmod 4 \pi^{0}\left(E^{2} P^{2}\right) p^{\prime \prime \prime}=0$. So we have $4 \overline{\bar{\eta}} p^{\prime \prime}=\eta^{2} \bar{\eta} p^{\prime \prime \prime}$ and $\pi^{4}\left(P^{8}\right)=\left\{\overline{\bar{\eta} p^{\prime \prime}}\right\}$ $\approx Z / 8$.

By the similar arguments to the above, we have the following

Proposition 4.7. $\pi^{k}\left(P^{8}\right)$ for $1 \leqq k \leqq 8$ is isomorphic to the corresponding group in the following table:

\begin{tabular}{|c||c|c|c|c|c|c|c|c|}
\hline$k$ & 1 & 2 & 3 & 4 & 5 & 6 & 7 & 8 \\
\hline$\pi^{k}\left(P^{8}\right)$ & $(2)^{2}$ & 0 & $(2)$ & $(8)$ & $(2)$ & $(2)$ & 0 & $(2)$ \\
\hline gen. & $\overline{\bar{\nu}^{2} p^{\prime \prime \prime}, \sigma p_{8}}$ & & $\overline{\overline{\nu p}_{6}}$ & $\overline{\bar{\eta} p^{\prime \prime}}$ & $\eta \bar{\eta} p^{\prime \prime \prime}$ & $\bar{\eta} p^{\prime \prime \prime}$ & & $p_{8}$ \\
\hline
\end{tabular}

Here $\overline{\bar{\eta} p^{\prime \prime}} \in\left\langle\bar{\eta} p^{\prime \prime}, \tilde{\eta}^{\prime} p, p^{\prime \prime \prime}\right\rangle$ and $4 \overline{\bar{\eta} p^{\prime \prime}}=\eta^{2} \bar{\eta} p^{\prime \prime \prime}$ 。

Remark 2. Hideaki Ōshima pointed out the following: 
Let $V_{n, k}$ denote the Stiefel manifold of $k$-frames in $R^{n}$. Then, according to [9], $\pi^{n-m}\left(P^{n}\right) \approx \pi_{N+m-n-1}\left(V_{N-1, n}\right)$, where $N=j 2^{s(n-1)}$ for a large integer $j$. So the group structures of $\pi^{2 n-m}\left(P^{2 n}\right)$ for $n \leqq 4$ are also obtained from the wellknown works of G. F. Paechter and C.S. Hoo.

By (4.5), (4.8) and (4.9), $\overline{\bar{\eta}}^{\prime} \tilde{i}^{\prime} \in\left\langle\overline{\bar{\eta}}, \tilde{\eta}^{\prime} p, i\right\rangle \supset\langle 2 \sigma, p, i\rangle \ni-\sigma \bmod \overline{\bar{\eta}} \pi_{7}\left(P^{6}\right)+$ $\pi^{0}\left(E^{6} P^{2}\right) i=\{2 \sigma\}$. So we have

$$
\overline{\bar{\eta}}^{\prime} \tilde{i}^{\prime} \equiv \sigma \bmod 2 \sigma
$$

\section{$\S 5$. Determination of $\left\{P^{8}, P^{8}\right\}$}

By use of (1.1) and Proposition 4.7, we have the following

Proposition 5.1. $\left\{P^{8}, E^{k} P^{2}\right\}$ for $0 \leqq k \leqq 6$ is isomorphic to the corresponding group in the following table:

\begin{tabular}{|c||c|c|c|c|c|c|c|}
\hline$k$ & 0 & 1 & 2 & 3 & 4 & 5 & 6 \\
\hline$\left\{P^{8}, E^{k} P^{2}\right\}$ & $(2)^{2}$ & $(2)$ & $(2)^{2}$ & $(2)^{2}$ & $(4)$ & $(2)$ & $(2)$ \\
\hline gen. & $i \bar{\nu}^{2} p^{\prime \prime \prime}, i \sigma p_{8}$ & $\widetilde{\overline{\nu p}}_{6}$ & $\tilde{\eta} \eta \bar{\eta} p^{\prime \prime \prime}, i \overline{\bar{\nu}}_{6}$ & $\tilde{\eta} \bar{\eta} p^{\prime \prime \prime}, i \overline{\bar{\eta} p^{\prime \prime}}$ & $\widetilde{\bar{\eta} p^{\prime \prime \prime}}$ & $i \bar{\eta} p^{\prime \prime \prime}$ & $p^{\prime \prime \prime}$ \\
\hline
\end{tabular}

Here $\widetilde{\overline{\nu p}}_{6} \in\left\langle i, 2 \iota, \overline{\overline{\nu p}}_{6}\right\rangle, \widetilde{\bar{\eta} p^{\prime \prime \prime}} \in\left\langle i, 2 \iota, \bar{\eta} p^{\prime \prime \prime}\right\rangle$ and $\widetilde{2 \bar{\eta} p^{\prime \prime \prime}}=i \eta \bar{\eta} p^{\prime \prime \prime}$.

Consider the following exact sequence induced from $(1.2)^{\prime \prime \prime}$ :

$$
\left\{E^{5} P^{2}, P^{2}\right\}^{\left(\tilde{\eta}^{\prime} p\right)^{*}}\left\{P^{6}, P^{2}\right\} \stackrel{i^{\prime \prime \prime *}}{\longleftarrow}\left\{P^{8}, P^{2}\right\} \stackrel{p^{\prime \prime \prime *}}{\longleftarrow}\left\{E^{6} P^{2}, P^{2}\right\} \stackrel{\left(\tilde{\eta}^{\prime} p\right)^{*}}{\longleftarrow}\left\{E P^{6}, P^{2}\right\} .
$$

Then, by Proposition 3.5, (4.3) and (4.9), $\left(\tilde{\eta}^{\prime} p\right)^{*}\left(i \nu \bar{p}_{4}\right)=i \nu^{2} p$ and $\left(\tilde{\eta}^{\prime} p\right)^{*}(i \eta)=$ $\left(\tilde{\eta}^{\prime} p\right)^{*}\left(i \nu^{2} p_{6}\right)=0$. We have also $\left(\tilde{\eta}^{\prime} p\right)^{*} \widetilde{\nu}_{4} \in\left\langle i, 2 \ell, \nu \bar{p}_{4}\right\rangle \circ \tilde{\eta}^{\prime} p \subset\left\langle i, 2 \iota, \nu^{2}\right\rangle p \ni \widetilde{\nu^{2}} p$ $\bmod i \sigma p$. So we have

$$
\widetilde{\nu^{2}} p_{8} \equiv 0 \bmod i \sigma p_{8}
$$

Remark 1. By the same arguments as the ones in the proof of Lemma 5.2, we have $\widetilde{\nu^{2}} p_{8}=0$.

Consider the following exact sequence induced from $(1.2)^{\prime}$ :

$$
\left\{P^{8}, E P^{2}\right\} \stackrel{(\tilde{\eta} p)_{*}}{\longrightarrow}\left\{P^{8}, P^{2}\right\} \stackrel{i_{*}^{\prime}}{\longrightarrow}\left\{P^{8}, P^{4}\right\} \stackrel{p_{*}^{\prime}}{\longrightarrow}\left\{P^{8}, E^{2} P^{2}\right\} \stackrel{(\tilde{\eta} p)_{*}}{\longrightarrow}\left\{P^{8}, E P^{2}\right\} .
$$

Then, by Proposition 5.1 and (4.7), $(\tilde{\eta} p)_{*} \cong_{\bar{\nu}}=\tilde{\eta} \overline{\bar{\nu}}_{6} \in \tilde{\eta}\left\langle\nu p_{6}, \tilde{\eta}^{\prime} p, p^{\prime \prime \prime}\right\rangle=\left\langle\tilde{\eta}, \nu p_{6}\right.$, $\left.\tilde{\eta}^{\prime} p\right\rangle p^{\prime \prime \prime} \supset\langle\tilde{\eta}, \nu, \eta p\rangle p^{\prime \prime \prime} \supset\langle\tilde{\eta}, \nu, \eta\rangle p_{8} \bmod \tilde{\eta} \pi^{0}\left(E^{3} P^{2}\right) p^{\prime \prime \prime}=0$. Since $p\langle\tilde{\eta}, \nu, \eta\rangle \subset\langle\eta, \nu$, $\eta\rangle=\nu^{2},\langle\tilde{\eta}, \nu, \eta\rangle \exists \widetilde{\nu^{2}} \bmod i \sigma$. So, by (5.1), $\tilde{\eta} \overline{\overline{\nu p}}_{6}=0$ or $i \sigma p_{8}$. 
Lemma 5.2. $\tilde{\eta} \overline{\overline{\nu p}}_{6}=0$ in $\left\{P^{s}, P^{2}\right\}$ and $i \sigma p_{8} \neq 0$ in $\left\{P^{8}, P^{4}\right\}$.

Proof. It suffices to prove $i \sigma p_{8} \neq 0$ in $\left\{P^{8}, P^{4}\right\}$. Consider the following EHP-sequence :

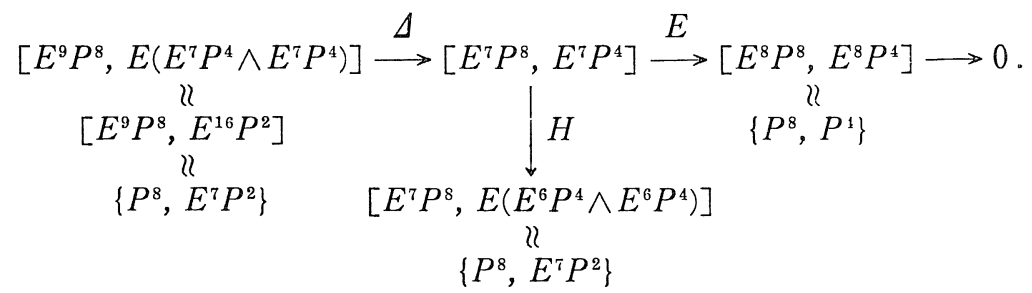

Here we have used the following: $P^{2} \wedge P^{2}=E P^{2} \cup_{2 c_{2}^{\prime}} C\left(E P^{2}\right)$ and so the 3-skeleton of $P^{2} \wedge P^{2}$ is stably equivalent to $E P^{2} \vee S^{3}$. Then, by use of $(1.2)^{\prime \prime \prime},\left\{P^{8}, E^{7} P^{2}\right\}$ $=\left\{i p_{8}\right\} \approx Z / 2 . \quad$ By inspecting Proposition 2.2 of $[10], \quad H\left(E^{7}\left(i^{\prime} i\right) \circ \sigma \circ E^{7} p_{8}\right)=$ $H\left(E^{7}\left(i^{\prime} i\right) \circ \sigma\right) \circ E^{7} p_{8}=E^{14} i \circ E^{7} p_{8} \neq 0$ since $H(\sigma)=\iota_{15}$. Here $\iota_{n}$ denotes the identity class of $S^{n}$. By Proposition 3.3 of [6], (5.16) of [10] and Lemma 3.1 of [8], $\Delta\left(E^{16} i \circ E^{9} p_{8}\right)=\Delta\left(E^{16} i\right) \circ E^{7} p_{8}=E^{7} i \circ \Delta\left(\iota_{17}\right) \circ E^{7} p_{8}=E^{7} i \circ\left(2 \sigma-E \sigma^{\prime}\right) \circ E^{7} p_{8}=E^{7} i \circ E \sigma^{\prime} \circ E^{7} p_{8}$, where $\sigma^{\prime}$ denotes the generator of the 2-component of $\left[S^{14}, S^{7}\right]$. So we have $H\left(\Delta\left(E^{16} i \circ E^{9} p_{8}\right)\right)=0$. Therefore $E^{7}\left(i^{\prime} i\right) \circ \sigma \circ E^{7} p_{8}$ is not in the image of $\Delta$. This completes the proof.

By Proposition 5.1 and Lemma 5.2, we have a short exact sequence

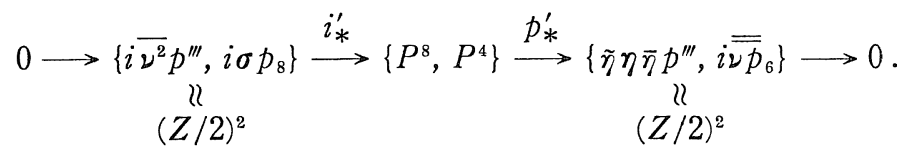

We define an element $\widetilde{\tilde{\eta} \eta \bar{\eta}} \in\left\{E^{6} P^{2}, P^{4}\right\}$ by $p^{\prime} \tilde{\eta} \eta \bar{\eta}=\tilde{\eta} \eta \bar{\eta}$, i. e.,

$$
\widetilde{\eta} \eta \bar{\eta} \in\left\langle i^{\prime}, \tilde{\eta} p, \tilde{\eta} \eta \bar{\eta}\right\rangle \text {. }
$$

By (2.3) and Proposition 4.7, there exists an element $\tilde{i \nu} \overline{\bar{p}}_{6} \in\left\{P^{8}, P^{4}\right\}$ and by Proposition 2.2 and Lemma 5.2, $2 \tilde{i} \overline{\bar{\nu}}_{6}=i^{\prime} \tilde{\bar{\nu}} \overline{\bar{p}}_{6}=0$. This leads us to the following

Proposition 5.3. $\left\{P^{8}, P^{4}\right\}=\left\{\widetilde{\tilde{\eta} \eta \bar{\eta}} p^{\prime \prime \prime}, \tilde{i} \overline{\bar{\nu}}_{6}, i \bar{\nu}^{2} p^{\prime \prime \prime}, i \sigma p_{8}\right\} \approx(Z / 2)^{4}$.

Lemma 5.4. i) $\quad \bar{\eta} \overline{\bar{\eta} p^{\prime \prime}}=0$. ii) $\tilde{\eta} \overline{\bar{\eta} p^{\prime \prime}}=0$. iii) $\overline{\bar{\eta}} \widetilde{\bar{\eta} p^{\prime \prime \prime}}= \pm 2 \overline{\bar{\eta} p^{\prime \prime}}$.

Proof. By Proposition 3.1, $\eta \bar{\eta} p^{\prime \prime}=\eta \circ 2 \bar{p}_{4}=0 . \quad$ By Proposition 4.7, $\eta \overline{\bar{\eta} p^{\prime \prime}} \in$ $\eta\left\langle\bar{\eta} p^{\prime \prime}, \tilde{\eta}^{\prime} p, p^{\prime \prime \prime}\right\rangle=\left\langle\eta, \bar{\eta} p^{\prime \prime}, \tilde{\eta}^{\prime} p\right\rangle p^{\prime \prime \prime} \subset \pi^{0}\left(E^{3} P^{2}\right) p^{\prime \prime \prime}=0 . \quad$ Therefore $\tilde{\eta} \overline{\bar{\eta}} \overline{p^{\prime \prime}} \in\langle i, 2 \iota, \eta\rangle$ $\overline{\bar{\eta} p^{\prime \prime}}=i\left\langle 2 \iota, \eta, \overline{\bar{\eta} p^{\prime \prime}}\right\rangle \subset i_{*} \pi^{2}\left(P^{8}\right)=0$. By Propositions 4.7 and 5.1, $2 \bar{\eta} \bar{\eta} p^{\prime \prime \prime}=\eta^{2} \bar{\eta} p^{\prime \prime \prime}=$ $4 \overline{\bar{\eta} p^{\prime \prime}} \in \pi^{4}\left(P^{8}\right)=\left\{\overline{\bar{\eta} p^{\prime \prime}}\right\} \approx Z / 8$. This completes the proof.

Remark 2. i) By Theorem 4.6, Proposition 4.7, (4.6) and Lemma 5.4. i), 
$\left\langle\eta, \nu, p_{8}\right\rangle \ni 0 \bmod \eta \pi^{4}\left(P^{8}\right)=0$. So, by Proposition 4.3, $\widetilde{i \nu} p_{8} \in\left\langle i^{\prime \prime}, \tilde{i} \bar{\eta}, i \nu\right\rangle p_{8}=$ $-i^{\prime \prime}\left\langle\tilde{i} \bar{\eta}, i \nu, p_{8}\right\rangle \supset i^{\prime \prime} \tilde{i}\left\langle\eta, \nu, p_{8}\right\rangle \ni 0 \bmod i^{\prime \prime} \pi_{8}\left(P^{4}\right) p_{8}=\left\{i^{\prime} \nu^{2} p_{8}, i \sigma p_{8}\right\}$. Therefore, by

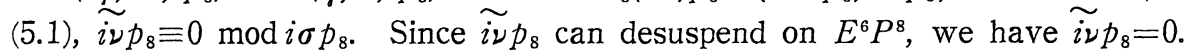

ii) By Proposition 4.7, $\tilde{i}^{\prime} \eta p_{8}=0$. So, by i), Proposition 4.4 and Remark 1 , $\gamma_{8} p_{8}=0$.

Conjecture. $\gamma_{2 n} p_{2 n}=0$ for all $n$.

Consider the following exact sequence induced from (1.2)" :

$$
\left\{P^{8}, E^{3} P^{2}\right\} \stackrel{(\tilde{i} \bar{\eta})_{*}}{\longrightarrow}\left\{P^{8}, P^{4}\right\} \stackrel{i_{*}^{\prime \prime}}{\longrightarrow}\left\{P^{8}, P^{6}\right\} \stackrel{p_{*}^{\prime \prime}}{\longrightarrow}\left\{P^{8}, E^{4} P^{2}\right\} \stackrel{(\tilde{i} \bar{\eta})_{*}}{\longrightarrow}\left\{P^{8}, E P^{4}\right\} .
$$

Then, by Propositions 2.5, 5.1 and Lemma 5.4, $(\tilde{i} \bar{\eta})\left(\tilde{\eta} \bar{\eta} p^{\prime \prime \prime}\right)=0$, $(\tilde{i} \bar{\eta})\left(i \overline{\bar{\eta}} \overline{p^{\prime \prime}}\right)=\tilde{i} \bar{\eta} \overline{\bar{\eta} p^{\prime \prime}}$ $=0$ and $(\tilde{i} \bar{\eta}) \widetilde{\bar{\eta} p^{\prime \prime \prime}}= \pm 2 \tilde{i} \overline{\bar{\eta}} \overline{p^{\prime \prime}}=i^{\prime} \tilde{\eta} \overline{\bar{\eta}} \overline{p^{\prime \prime}}=0$. So we have a short exact sequence

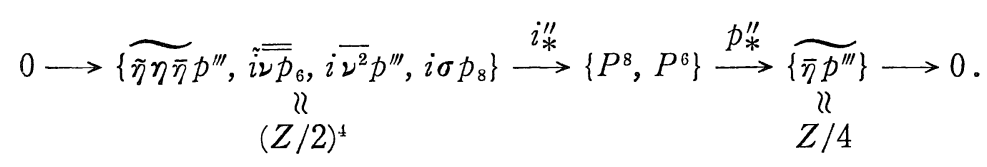

We define an element $\widetilde{\bar{\eta} p^{\prime \prime \prime}} \in\left\{P^{8}, P^{6}\right\}$ by $p^{\prime \prime} \widetilde{\bar{\eta} p^{\prime \prime \prime}}=\widetilde{\bar{\eta} p^{\prime \prime \prime}}$, i. e.,

$$
\widetilde{\bar{\eta}}^{\prime \prime \prime}{ }^{\prime} \in\left\langle i^{\prime \prime}, \tilde{i} \bar{\eta}, \widetilde{\bar{\eta} p^{\prime \prime \prime}}\right\rangle \text {. }
$$

By use of $(1.2)^{\prime \prime \prime}$ and Proposition 5.1, we have a short exact sequence

$$
0 \longrightarrow\left\{P^{8}, P^{6}\right\} \stackrel{i_{*}^{\prime \prime \prime}}{\longrightarrow}\left\{P^{8}, P^{8}\right\} \stackrel{p_{*}^{\prime \prime \prime}}{\longrightarrow}\left\{p^{\prime \prime \prime}\right\} \longrightarrow 0 .
$$

Since $\iota_{8}^{\prime}$ is of order 16 , there exists an element of order 8 in $\left\{P^{8}, P^{6}\right\}$ which is mapped onto $2 \iota_{8}^{\prime}$ by $i_{*}^{\prime \prime \prime}$. So this element must be $\widetilde{\bar{\eta} p^{\prime \prime \prime}}{ }^{\prime}$ modulo elements of order 2. Therefore we have $2 c_{8}^{\prime} \equiv i^{\prime \prime \prime} \bar{\eta} p^{\prime \prime \prime} \bmod$ some elements of order 2$\}$. By Theorem 4.6, $\overline{\bar{\eta}}\left(4 \widetilde{\bar{\eta} p^{\prime \prime \prime}}\right)=8 \overline{\bar{\eta}}^{\prime}=\overline{8 \sigma} p^{\prime \prime \prime}$. This leads us to

$$
4 \overline{\bar{\eta}} \widetilde{\bar{\eta} p^{\prime \prime \prime}}=\overline{8 \sigma} p^{\prime \prime \prime} .
$$

Lemma 5.5.

$$
\bar{\eta}^{\prime} \tilde{\eta} \eta \bar{\eta} \equiv \overline{8 \sigma} \bmod \pi_{8}\left(S^{0}\right) p \text {. }
$$

Proof. By (2.2) and (5.2), $\bar{\eta}^{\prime} \tilde{\eta} \eta \bar{\eta} \in\langle\bar{\eta}, \tilde{\eta} p, \tilde{\eta} \eta \bar{\eta}\rangle \subset\langle\bar{\eta}, \tilde{\eta} \eta, \eta \bar{\eta}\rangle \bmod \bar{\eta}\left\{E^{6} P^{2}\right.$, $\left.P^{2}\right\}+\pi_{5}\left(S^{0}\right)(\eta \bar{\eta})=\pi_{8}\left(S^{0}\right) p$. By (4.9) and Proposition 4.1. ii), $8 \sigma=4 \overline{\bar{\eta}} \tilde{\eta}^{\prime} \equiv \bar{\eta}^{\prime} \tilde{\eta} \eta^{2}$ $\bmod \bar{\eta}\left(i \nu^{2}\right)=0$. So, by $(2.2)$ and (4.1), $8 \sigma=\bar{\eta}^{\prime} \tilde{\eta} \eta^{2} \in\left\langle\bar{\eta}, \tilde{\eta} p, \tilde{\eta} \eta^{2}\right\rangle \subset\left\langle\bar{\eta}, \tilde{\eta} \eta, \eta^{2}\right\rangle$ $\bmod \bar{\eta} \pi_{7}\left(P^{2}\right)+\pi_{5}\left(S^{0}\right) \eta^{2}=0$. By (2.2), (4.1) and (5.2), $\bar{\eta}^{\prime} \tilde{\eta} \eta \bar{\eta} i \in\left\langle\bar{\eta}, \tilde{\eta} p, \tilde{\eta} \eta^{2}\right\rangle \ni \bar{\eta}^{\prime} \tilde{\eta} \eta^{2}$ $\bmod \bar{\eta} \pi_{7}\left(P^{2}\right)+\pi^{0}\left(E^{2} P^{2}\right) \tilde{\eta} \eta^{2}=0$. Therefore $\bar{\eta}^{\prime} \tilde{\eta} \eta \bar{\eta} i=\bar{\eta}^{\prime} \tilde{\eta} \eta^{2}=8 \sigma$. This completes the proof. 
Remark 3. According to [7], the equality $\left\langle\bar{\eta}, \tilde{\eta} \eta, \eta^{2}\right\rangle=8 \sigma$ holds on $S^{5}$.

By Lemma 5.5 and (4.6), $\overline{\bar{\eta}}\left(i^{\prime \prime} \tilde{\eta} \eta \bar{\eta} p^{\prime \prime \prime}\right)=\overline{8 \sigma} p^{\prime \prime \prime}$. By (2.4) and Theorem 4.6, $\overline{\bar{\eta}}\left(i^{\prime \prime} \tilde{i \nu \overline{\bar{p}}_{6}}\right)=\nu \overline{\bar{\nu}}_{6} \neq 0$. So, by (5.4), we have

$$
4 \widetilde{\bar{\eta} p^{\prime \prime \prime}} \equiv i^{\prime \prime} \tilde{\eta} \eta \bar{\eta} p^{\prime \prime \prime} \bmod \left\{i \overline{\nu^{2}} p^{\prime \prime \prime}, i \sigma p_{8}\right\}
$$

and

$$
2 \iota_{8}^{\prime} \equiv i^{\prime \prime \prime} \widetilde{\bar{\eta} p^{\prime \prime \prime}} \bmod \left\{i^{\prime \prime} \tilde{i \nu \overline{\bar{p}}_{6}}, i \bar{\nu}^{2} p^{\prime \prime \prime}, i \sigma p_{8}\right\} .
$$

Therefore (5.5) and (5.6) lead us to the following

Theorem 5.6.

i) $\left\{P^{8}, P^{6}\right\}=\left\{\widetilde{\bar{\eta}}^{\prime \prime \prime \prime}, i^{\prime \prime} i \overline{\bar{\nu}}_{6}, i \bar{\nu}^{2} p^{\prime \prime \prime}, i \sigma p_{8}\right\} \approx Z / 8 \oplus(Z / 2)^{3}$.

ii) $\left\{P^{8}, P^{8}\right\}=\left\{\epsilon_{8}^{\prime}, i^{\prime \prime} i \overline{\overline{\nu p}}_{6}, i \bar{\nu}^{2} p^{\prime \prime \prime}, i \sigma p_{8}\right\} \approx Z / 16 \oplus(Z / 2)^{3}$.

We put $\alpha=i \prime i \overline{\bar{\nu}}_{6}, \beta=i \bar{\nu}^{2} p^{\prime \prime \prime}$ and $\gamma=i \sigma p_{8}$. Then $\alpha^{2}=\beta^{2}=\gamma^{2}=0, \alpha \beta=\beta \alpha=0$, $\beta \gamma=\gamma \beta=0$ and $\alpha \gamma=\gamma \alpha=0$. So we have the following

Corollary.

$$
\xi\left(P^{8}\right) \approx(Z / 16)^{*} \times(Z / 2)^{3}
$$

\section{References}

[1] Atiyah, M.F., Thom complexes, Proc. London Math. Soc., 11 (1961), 291-310.

[2] Liulevicius, A., A theorem in homological algebra and stable homotopy of projective spaces, Trans. Amer. Math. Soc., 109 (1963), 540-552.

[3] Mahowald, M., The metastable homotopy of $S^{n}$, Mem. Amer. Math. Soc., 72, 1967.

[4] Mukai, J., Stable homotopy of some elementary complexes, Mem. Fac. Sci. Kyushu Univ., A 20 (1966), 266-282.

[5] — On the stable homotopy of a $Z_{2}$-Moore space, Osaka J. Maih., 6 (1969), 63-91.

[6] - A characterization of the Kahn-Priddy map, to appear in Advanced Studies in Pure Math. 1986.

[7] Oka, S., Homotopy of the exceptional Lie group $G_{2}$, to appear in Proc. Edinburgh Math. Soc. 1986.

[8] Ōshima, H., A remark on unstable James numbers, Osaka J. Math., 21 (1984), 765-772.

[9] - On some cohomotopy of projective spaces. Preprint.

[10] Toda, H., Composition methods in homotopy groups of spheres, Ann. of Math. Studies, 49, Princeton, 1962.

[11] - Order of the identity class of a suspension space, Ann. of Math., 78 (1963), 300-325. 
